\title{
Marked increase in the number and variety of mitochondrial DNA rearrangements in aging human skeletal muscle
}

\author{
S. Melov, J. M. Shoffner, A. Kaufman and D. C. Wallace* \\ Department of Genetics and Molecular Medicine, School of Medicine, Emory University, 1462 Clifton Rd, NE \\ Atlanta, GA 30322, USA
}

Received June 29, 1995; Revised and Accepted September 13, 1995

\begin{abstract}
Several reports have shown that individual mitochondrial DNA (mtDNA) deletions accumulate with age. However, the overall extent of somatic mtDNA damage with age remains unclear. We have utilized full-length PCR to concurrently screen for multiple mtDNA rearrangements in total DNA extracted from skeletal muscle derived from physiologically normal individuals $(n=35)$. This revealed that both the number and variety of mtDNA rearrangements increases dramatically between young and old individuals $(P<0.0001)$. We further examined the mtDNA from both the younger and older subjects by Southern blot analysis and observed an age-related increase in mtDNA(s) comparable in size to mtDNA products unique to patients with known mtDNA deletions. These data imply that a wide spectrum of mtDNA rearrangements accumulate in old individuals, which correlates with the marked age related decrease in OXPHOS capacity observed in post-mitotic tissues.
\end{abstract}

\section{INTRODUCTION}

The molecular basis for this OXPHOS decline is unknown. However, it has been hypothesized that the decline is the product of the accumulation of somatic mitochondrial DNA (mtDNA) mutations in post-mitotic tissues. The accumulation of mtDNA rearrangements has been confirmed in a variety of post-mitotic tissues by quantitating the levels of specific mtDNA deletions.

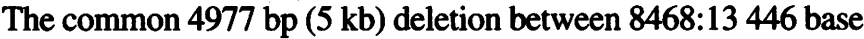
pairs (bp) has been most extensively studied $(9,10)$ and shown to increase with age in heart (11), skeletal muscle (9) and brain $(12,13)$. In the human brain, the amount of the $5 \mathrm{~kb}$ deletion has been found to rise rapidly after 75 years of age reaching levels in excess of $11-12 \%$ in the basal ganglia and $2-3 \%$ in the cerebral cortex, while remaining very low in the cerebellum (13). Other pathologic mtDNA mutations have been shown to increase concurrently with the $5 \mathrm{~kb}$ deletion, including the $7.4 \mathrm{~kb}$ deletion and $10.4 \mathrm{~kb}$ insertion-deletion in brain and heart $(11,14)$ as well as the pathogenic point mutations in tRNALeu(UUR) at $3243 \mathrm{bp}$ and tRNA ${ }^{\text {Lys }}$ at 8344 bp which cause the MELAS and MERRF syndromes, respectively (15-17).

While it is now well established that somatic mtDNA mutations accumulate with age, the levels of any one mtDNA mutation are too low to cause significant tissue pathology. In the multisystem disorders caused by mtDNA rearrangements, such as the ocular myopathies including the Kearns-Sayre syndrome (KSS) and chronic progressive external opthalmoplegia (CPEO), the common deletion can accumulate to $>50 \%$ of the patient's muscle mtDNA before debilitating muscle pathology becomes apparent (18). This has led to the proposal that aging is not the product of the accumulation of just one type of mtDNA damage, but rather is the sum total of a large number of mtDNA mutations which accumulate concurrently over time. Thus, the assessment of just one type of mtDNA mutation represents the 'tip of the iceberg' $(9,19)$. Molecular histology studies have shown that in KSS and CPEO muscle, the mutant mtDNA are regionally localized along muscle fibers $(20,21)$. Additionally, in aging muscle fibers, different mtDNA mutations are localized in different regions of the fiber (22), suggesting that individual mutations arise randomly along the muscle fibers with age and are subsequently clonally amplified (19).

To substantiate the 'tip of the iceberg' hypothesis, a simple method is needed for the collective assessment of all mtDNA rearrangements in an aging tissue. Only by showing directly that a significant proportion of the mtDNA in older tissues are mutant will it be possible to argue that somatic mtDNA mutations are a major factor in the decline of function of post-mitotic tissues. In this report, we present experiments which permit concurrent visualization of multiple mtDNA rearrangements. Application of these methods to aging human skeletal muscle shows that a wide variety of mtDNA rearrangements accumulate in skeletal muscle by age 75 .

\section{MATERIALS AND METHODS}

Human subjects

Protocols and consent forms were approved by the Emory University Human Investigations Committee. Normal healthy volunteers were recruited and after informed consent were

${ }^{*}$ To whom correspondence should be addressed 
subjected to a family history assessment, mini mental status testing, neurological examination, blood and urine organic and amino acids analysis, quantitation of carnitine and acyl-carnitine in blood and urine and a quadriceps muscle biopsy. Skeletal muscle biopsies were also obtained from two CPEO patients in which $39 \%$ (patient 1) and $52 \%$ (patient 2) of their muscle mtDNA contained the common $5 \mathrm{~kb}$ deletion as determined by BamHI restriction and Southern blot analysis. Total muscle genomic DNA was isolated from $50-100 \mathrm{mg}$ of the skeletal muscle by homogenization and lysis with SDS (23) followed by serial extraction with phenol, phenol-chloroform (1:1), chloroform and ethanol precipitation.

\section{Long extension PCR (LX-PCR)}

Long extension-PCR permits amplification of the entire mitochondrial genome from opposing primers located in the cytochrome $b$ gene (24). Using LX-PCR with a 10 min extension time permits amplification of the full-length mitochondrial genome with high efficiency. Long extension-PCR using a 5 min extension time permits some amplification of full-length molecules, but preferentially amplifies rearranged mtDNA molecules in which the primers are brought closer together.

The LX-PCR employed the primers FLFor (15 148-15 174 bp) and FLRev (14 841-14 $816 \mathrm{bp}$ ) (25) and the Expand ${ }^{\mathrm{TM}}$ Long Template PCR system (Boehringer Mannheim, Indianapolis, IN) to generate a $16231 \mathrm{bp}$ molecule from normal mtDNA. A biphasic hot-start amplification is employed using $50 \mu \mathrm{l} \mathrm{PCR}$ gems $^{\mathrm{TM}}$ (Perkin-Elmer, Foster City, CA). The bottom phase of the PCR $(30 \mu \mathrm{l})$ contained $300 \mu \mathrm{M}$ dNTPs and $0.2 \mu \mathrm{M}$ FLFor and FLRev (final concentrations) in thin-walled microamp tubes (US Biochemical, Cleveland, OH). A PCR gem ${ }^{\mathrm{TM}}$ (Perkin-Elmer) was placed on top of the bottom phase, melted at $80^{\circ} \mathrm{C}$ for $5 \mathrm{~min}$ and the top phase added. The top phase consisted of $1 \times$ PCR buffer for the reaction (buffer 2 from the kit), $1.75 \mathrm{U}$ of enzyme and 100 ng of genomic DNA as target. The PCR profiles consisted of initial 2 min denaturation at $94^{\circ} \mathrm{C}$, followed by two-step PCR for 35 cycles using 5 or $10 \mathrm{~min}$ annealing/extension at $68^{\circ} \mathrm{C}, 10 \mathrm{~s}$ denaturation at $94^{\circ} \mathrm{C}$ and a final extension of $10 \mathrm{~min}$ at $72^{\circ} \mathrm{C}$ in a 9600 thermal cycler (Perkin-Elmer).

Some variation was observed in the yield of full-length mtDNA PCR product in young subjects when amplifying from a constant amount of genomic DNA (Figs 1-5). However, this variation does not correlate with the slight variation in the common mtDNA intensities of linearized species in Southern blots of the same individuals (Fig. 6). Hence, the variation in LX-PCR yield probably refelcts variation in the PCR reaction rather than large fluctuations in the ratio of mtDNA to nuclear DNA samples.

\section{Field inversion gel electrophoresis (FIGE)}

A $5 \mu$ aliquot of the PCR was electrophoresed on $0.7 \%$ agarose gels $\left(266 \mu \mathrm{g} \mathrm{l}^{-1}\right.$ ethidium bromide) in $1 \times$ Tris-Acetate-EDTA (TAE) for 7-14 h using FIGE. Field inversion gel electrophoresis conditions consisted of $0.5 \mathrm{~s}$ forward direction and $0.25 \mathrm{~s}$ reverse direction at $4^{\circ} \mathrm{C}$ using $2.5 \mathrm{~V} / \mathrm{cm}$. FIGE gels were photographed on a UV transilluminator then denatured, neutralized and Southern blotted onto Hybond N (Amersham, Arlington Heights, IL) (26). Gel purified full-length PCR mtDNA probes were used for Southern hybridization, following labeling using the 'DIG DNA Labeling and Detection kit ${ }^{\mathrm{TM}}$, (Boehringer Mannheim).

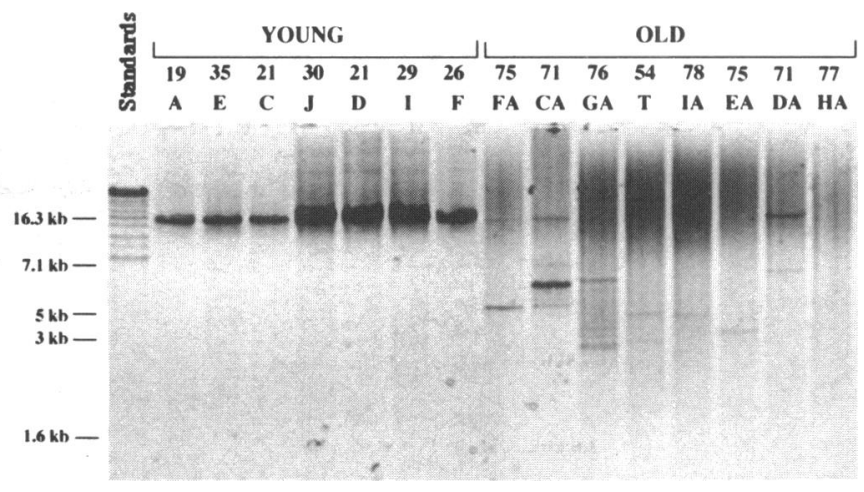

Figure 1. Ethidium bromide stained gels of LX-PCR using DNA from young and old individuals with 10 min extension times using FIGE. Each lane is designated by a letter or group of letters to differentiate between individuals. Numbering refers to the individual's age in years. Molecular size markers shown are the BRL high molecular weight standards

Hybridization and wash conditions were carried out under conditions of high stringency. Molecular weight standards were Lambda HindIII (Sigma, St Louis, MO), 1 kb Ladder (Gibco/ BRL, Gaithersburg, MD) and High Molecular Weight standards (Gibco/BRL).

\section{Southern blotting analysis}

Genomic DNA $(2.5 \mu \mathrm{g})$ was either left unrestricted or cut with EcoRV or BamHI, concentrated by ethanol precipitation and electrophoresed on $0.8 \%$ agarose slab gels $[1 \times$ Tris-borateEDTA (TBE)]. The completed gels were denatured, neutralized, Southern blotted onto nylon membranes (Zeta-Probe, Bio-Rad, Hercules, CA) and hybridized with ${ }^{32}$ P-labeled HeLa mtDNA (27).

\section{Statistical analysis}

Excluding full-length mtDNA, the number of LX-PCR products detected by ethidium bromide staining were tabulated and averaged for young and old subjects. The mean number of products were calculated and a comparison between the young and old subjects was carried out by a two tailed non-parametric Wilcoxon $t$-test.

\section{RESULTS}

\section{Increased mtDNA rearrangements in old subjects detected by LX-PCR}

The mtDNA structures of skeletal muscle from young and old subjects were analyzed by LX-PCR. Using a 10 min extension time, DNA from young subjects gave predominantly full-length mtDNA PCR products, while DNA from old subjects gave reduced or no full-length product. Moreover, the DNA from older subjects gave multiple additional lower molecular weight products, while those from younger subjects did not (Figs 1 and 2). This age-related difference in PCR products was accentuated using a 5 min extension time which limits the efficiency of full-length mtDNA amplification. In this case, full length molecules were faintly seen in young subjects, but absent in old subjects. Furthermore, old subjects had a heterogeneous array of 


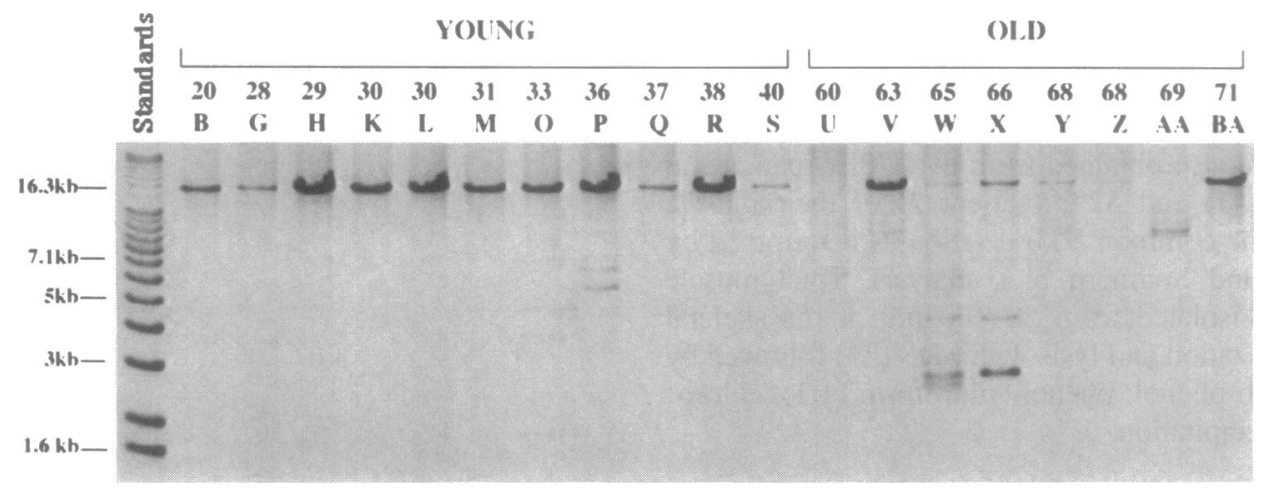

Figure 2. Ethidium bromide stained gels of LX-PCR using DNA from young and old individuals with 10 min extension times using FIGE. Each lane is designated by a letter or group of letters to differentiate between individuals. Numbering refers to the individual's age in years. Molecular size markers shown are BRL $1 \mathrm{~kb}$ ladder and high molecular weight standards.

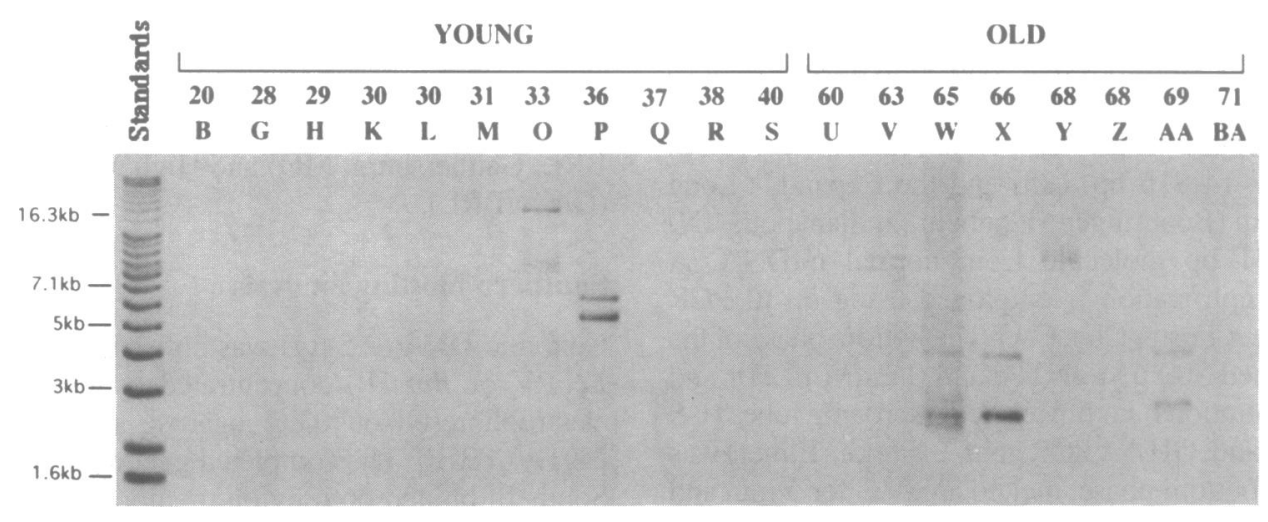

Figure 3. Ethidium bromide stained gels of LX-PCR using DNA from young and old individuals with 5 min extension times using FIGE. Nomenclature is identical to that described in the legends for Figures 1 and 2. Molecular size markers shown are the BRL 1 kb ladder.

smaller PCR products, which were virtually absent in young subjects, with the exception of individual P (Figs 3 and 4). To confirm that all of the LX-PCR products observed were of mtDNA origin, the DNA fragments from the 5 min extension LX-PCR shown in Figure 4 were denatured, transferred to nylon filters by Southern blotting and hybridized with a digoxigeninlabeled full-length PCR mtDNA probe. All of the PCR products seen by ethidium bromide staining were also found to hybridize to the mtDNA probe confirming that they are derived from the mitochondrial genome (Fig. 5).

Comparison of the mobility of the smaller mtDNA products with the molecular weight standards revealed that no single mtDNA rearrangement was common to all old subjects, nor did one mtDNA product tend to predominate. Rather, each individual had a distinctive array of rearrangements. For the $10 \mathrm{~min}$ extension LX-PCRs, the size of the products suggested that the rearrangements removed between 4 and $14 \mathrm{~kb}$ of the mtDNA. To quantitatively confirm the difference in the mtDNA structures between young and old subjects, the number of smaller molecular weight products seen in the ethidium bromide-stained 5 min extension LX-PCR were counted for each subject. The average number of rearranged molecules in young muscle was 0.33 per individual, while that for older individuals was 2.75 (Table 1). This difference was highly statistically significant $(P<0.0001)$.

\section{Analysis of mtDNA modification by direct Southern blot analysis}

To further analyze the structure of mtDNA in the muscle of old versus young subjects, we examined both restricted and unrestricted mtDNA by electrophoresis, Southern blotting and hybridization with a radioactive total mtDNA probe. The muscle DNA of five young subjects ( $R, D, B, O$ and $C)$, which exhibited no rearranged molecules by LX-PCR, were found to include linear molecules migrating at $16.5 \mathrm{~kb}$, plus a variety of other more slowly migrating species (Region II, Fig. 6). In contrast, the muscle DNA from four older subjects (V, HA, T and BA), which exhibited multiple rearranged mtDNA by LX-PCR, gave a Southern blot pattern similar to that of the younger subjects, with the exception that all four older muscle mtDNA exhibited an additional mtDNA species which migrated more rapidly than the conformationally complex mtDNA (Region I, Fig. 6). This mtDNA species corresponded in mobility to a unique mtDNA species in the CPEO patients (Region I, Fig. 6). 


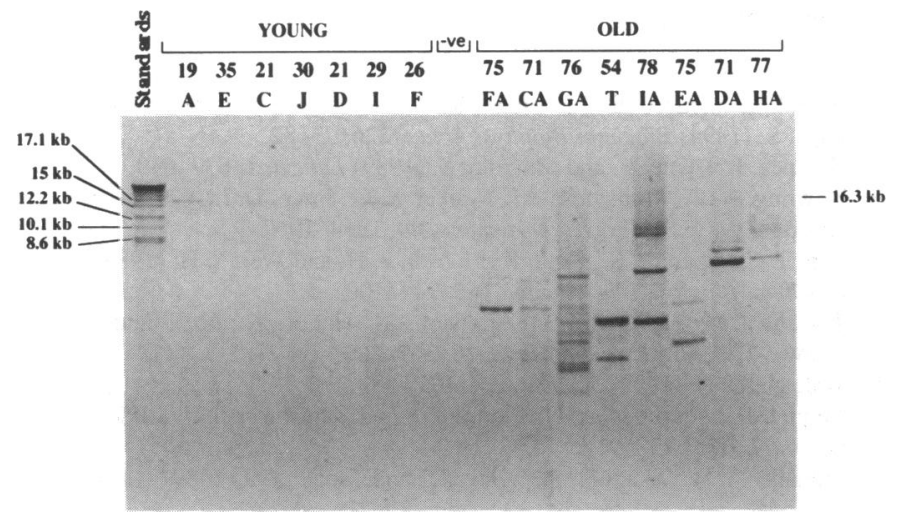

Figure 4. Ethidium bromide stained gels of LX-PCR using DNA from young and old individuals with 5 min extension times using FIGE. Nomenclature is identical to that described in the legends for Figures 1 and 2. -ve refers to the negative control used in each PCR experiment, that is no template DNA was added in the PCR. Molecular size markers shown are the BRL high molecular weight standards.

Table 1. Detectable dmtDNA by LX-PCR using 5 min extensions

\begin{tabular}{llllll}
\hline \multicolumn{2}{l}{ Young (19-40 years) } & & \multicolumn{3}{l}{ Old (54-78 years) } \\
Age (years) & Individual & dmtDNA & Age (years) & Individual & dmtDNA \\
\hline 19 & A & - & 54 & T & $*$ \\
20 & B & - & 60 & U & - \\
21 & C & - & 63 & V & $*$ \\
21 & D & - & 65 & W & $* *$ \\
24 & E & - & 66 & X & $*$ \\
26 & F & - & 68 & Y & $*$ \\
28 & G & - & 68 & Z & - \\
29 & H & - & 69 & AA & $*$ \\
29 & I & - & 71 & BA & $* *$ \\
30 & J & - & 71 & CA & $* *$ \\
30 & $\mathrm{~K}$ & - & 71 & DA & $* *$ \\
30 & L & $*$ & 75 & EA & $*$ \\
31 & M & - & 75 & FA & $* *$ \\
32 & $\mathrm{~N}$ & $*$ & 76 & GA & $* * *$ \\
33 & O & $*$ & 77 & HA & $* *$ \\
36 & P & $*$ & 78 & IA & $* *$ \\
37 & Q & - & & & \\
38 & $\mathrm{R}$ & - & & & \\
40 & $\mathrm{~S}$ & - & & & \\
\hline
\end{tabular}

Mean no. of dmtDNA detected in young individuals $=0.33 \mathrm{dmtDNA} /$ individual. Mean no. of dmtDNA detected in old individuals $=2.75 \mathrm{dmtDNA} /$ individual. *1-2 dmtDNA; **3-6 dmtDNA; ***> 7 dmtDNA.

$\mathrm{NB}$, individual $\mathrm{N}$ not shown in Figures $1-4$.

BamHI restriction (unique restriction site at $14258 \mathrm{bp}$ ) of total DNA from the various aged volunteers and two patients greatly simplified the patterns seen in the autoradiograms of the Southern blots. The CPEO patient mtDNA were converted to two

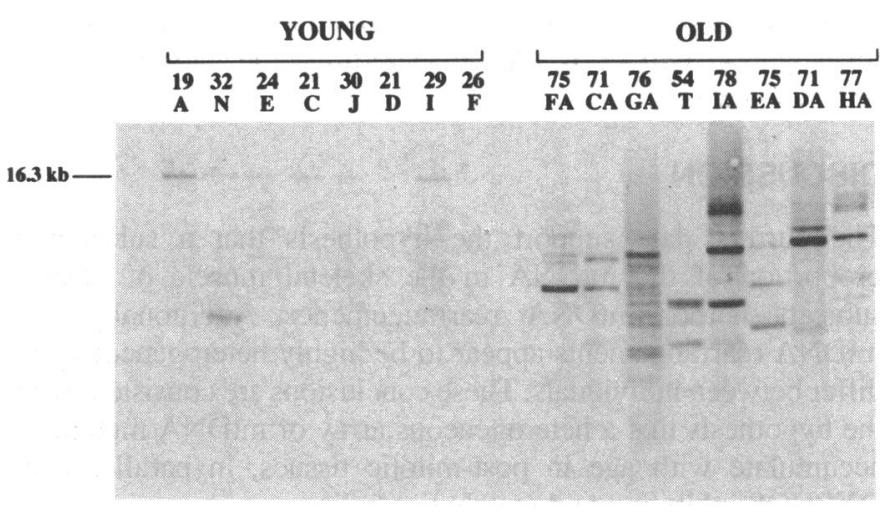

Figure 5. Southern blot of reactions from the 5 min extension LX-PCR shown in Figure 4 probed with total DIG-labeled mtDNA and detected by the DIG DNA Labeling and Detection kit ${ }^{\mathrm{m}}$. The individual designations and ages of the young and old individuals are shown above the lanes in years. Individual $\mathrm{N}$ is not shown in Figures 1-4.

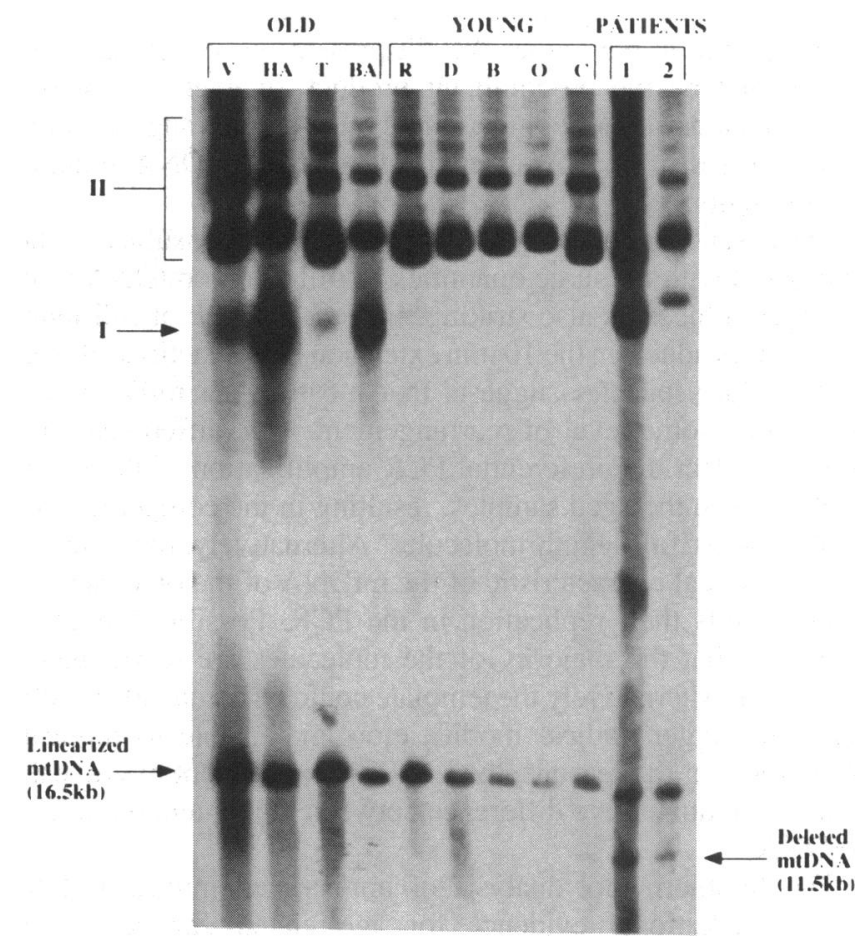

Figure 6. Southern blot of unrestricted total DNA from young and old individuals and CPEO patients using linear electrophoresis, probed with total 32P-labeled mtDNA and detected by autoradiography. Linearized and deleted mtDNA are indicated for normal individuals and patients, respectively. Among the young subjects, individuals $B, C$ and $D$ are 20-21 years old and $O$ and $R$ are 30 and 36 years old. For the older subjects, individual $\mathrm{T}$ is $54, \mathrm{~V}$ is $63, \mathrm{BA}$ is 71 and HA is 77 . Patients 1 and 2 are 47 and 65 years old, respectively.

fragments: $16.5 \mathrm{~kb}$ full length linear molecules and $11.5 \mathrm{~kb}$ deleted linear molecules. The various age volunteer mtDNA were reduced to a prominent $16.5 \mathrm{~kb}$ full length linear molecule (data not shown). These data indicate that most if not all of the slower migrating forms in Region II of the gel (Fig. 6) are conformational variants of the full length and/or rearranged molecules, and that 
an additional mtDNA species, 'I' accumulates with age. Further investigation will be needed to determine the nature of these mtDNA species.

\section{Discuseion}

The current data support the hypothesis that a substantial proportion of the mtDNA in the skeletal muscle of elderly subjects harbor mtDNA rearrangements. Additionally, the mtDNA rearrangements appear to be highly heterogeneous and differ between individuals. These conclusions are consistent with the hypothesis that a heterogeneous array of mtDNA mutations accumulate with age in post-mitotic tissues, in parallel with OXPHOS inhibition and metabolic decline.

While the LX-PCR procedure is not quantitative, it reveals a dramatic qualitative difference between the mtDNA of young and old muscle. Not only do the muscles of older subjects have many more rearranged $\mathrm{mtDNA}$ PCR products, they frequently lack any full-length mtDNA PCR products. The great heterogeneity of the smaller LX-PCR products in the older samples is striking. The common $4977 \mathrm{bp}(5 \mathrm{~kb})$ deletion observed in one-third to one-half of KSS and CPEO patients $(6,19)$ has been shown to increase with age in multiple studies. Yet the present analysis suggests that it may not be the predominant product, or even a consistent product. Hence quinititating a sing/e mtBNA rearrangement may result in a distosted perspective of, somatic miDNA mutation accumulation.

The absence of full-length PCE products in older subjects when compared with the large quantities of full-length mtDNA in the younger subjects is also striking. Indeed, the lack of full-length LX-PCR products in the 10 min extension PCR reactions of many of the older muscles suggests that most of the mtDNA have undergone some level of rearrangement. This difference might merely reflect the preferential PCR amplification of the shorter molecules in the aged samples, resulting in the competitive loss of the longer full-length molecules. Alternatively, there may be some physical characteristic of the mtDNA of the older subjects which limits their replication in the PCR. Possible limitations could be that the majority of the molecules are rearranged or catenated. Alternatively the template could be damaged, possibly through oxygen radical modification and unable to replicate. Regardless of the reasons, the LX-PCR experiment has revealed a dramatic qualitative difference between young and old muscle mtDNA.

The Southern blot analysis of unrestricted muscle mtDNA provide additional evidence for age-related changes in the mtDNA. However, the precise identity of these alterations is unclear at this time.

The age-related accumulation of mtDNA damage may be a general phenomenon in multicellular animals. Mitochondrial DNA mutations have been found to accumulate with age in post-mitotic somatic tissues of animals as diverse as mouse (28), rat (29) Maccaca mulatta (30) and Caenorhabditis elegans (31). One likely cause for this damaged mtDNA could be oxygen radicals generated as a toxic byproduct of OXPHOS itself $(6,19,32-34)$.

\section{REFERENCES}

1 Boffoli, D., Scacco, S. C., Vergari, R., Solarino, G., Santacroce, G. and Papa, S. (1994) Biochim. Biophys. Acta, 1226, 73-82.

2 Trounce, I., Byme, E. and Marzuki, S. (1989) Lancet, 1, 637-639.

3 Bowling, A. C., Mutisya, E. M., Walker, L. C., Price, D. L., Cork; L. C. and Beal, M. F. (1993) J. Neurochem., 60, 1964-1967.

4 Yen, T. C., Chen, Y. S., King, K. L., Yeh, S. H. and Wei, Y. H. (1989) Biochem. Biophys. Res. Comm., 165, 944-1003.

5 Takasawa, M., Hayakawa, M., Sugiyama, S., Hattori, K., Ito, T. and Ozawa, T. (1993) Exp. Genontol, 28, 269-280.

6 Wallace, D. C. (1992) Science, 256, 628-632.

7 Miquel, J., Economos, A. C., Fleming, J. and Johnson Jr, J. E. (1980) Exp. Gerontol, 15, 575-591.

8 Linnane, A. W., Marzuki, S., Ozawa, T. and Tanaka, M. (1989) Lancet, 1, 642-645.

9 Cortopassi, G. A., Shibata, D., Soong, N. W. and Armheim, N. (1992) Proc. Natl Acad. Sci. USA, 89, 7370-7374.

10 Cortopassi, G. A. and Amheim, N. (1990) Nucleic Acids Res., 18, 6927-6933.

11 Corral-Debrinski, M., Shoffner, J. M., Lott, M. T. and Wallace, D. C. (1992) Mutat. Res., 275, 169-180.

12 Corral-Debrinski, M., Horton, T., Lott, M. T., Shoffiner, J. M., Beal, M. F. and Wallace, D. C. (1992) Nature Genet., 2, 324-329.

13 Soong, N. W., Hinton, D. R., Cortopassi, G. and Amheim, N. (1992) Nature Genet., 2, 318-323.

14 Hayakawa, M., Hattori, K., Sugiyama, S. and Ozawa, T. (1992) Biochem. Biophys. Res. Comm., 189, 979-985.

15 Zhang, C., Linnane, A. W. and Nagley, P. (1993) Biochem. Biophys. Res. Comm., 195, 1104-1110.

16 Munscher, C., Muller-Hocker, J. and Kadenbach, B. (1993) Biol. Chem. Hoppe Seyler, 374, 1099-1104.

17 Munscher, C., Rieger, T., Muller-Hocker, J. and Kadenbach, B. (1993) FEBS Lett., 317, 27-30.

18 Wallace, D. C. (1994) J. Bioenerg. Biomembr., 26, 241-250.

19 Wallace, D. C., Richter, C., Bohr, V. A., Cortopassi, G., Kadenbach, B., Linn, S., Linnane, A. W. and Shay, J. W. (1995) In Esser, K. and Martin, G. M. (eds), Molecular Aspects of Aging. John Wiley \& Sons, in press.

20 Shoubridge, E. A., Karpati, G. and Hastings, K. E. (1990) Cell, 62, 43-49.

21 Tokunaga, M., Mita, S., Sakuta, R., Nonaka, I. and Araki, S. (1993) Ann. Neurol., 33, 275-280.

22 Muller-Hocker, J., Seibel, P., Schneiderbanger, K. and Kadenbach, B. (1993) Virch. Arch. A. Pathol. Anat. Histopathol., 422, 7-15.

23 Shoffner, J. M., Lott, M. T., Lezza, A. M., Seibel, P., Ballinger, S. W. and Wallace, D. C. (1990) Cell, 61, 931-937.

24 Cheng, S., Higuchi, R. and Stoneking, M. (1994) Nature Genet., 7, 350-351.

25 Anderson, S., Bankier, A. T., Barrell, B. G., de Bruijn, M. H., Coulson, A. R., Drouin, J., Eperon, I. C., Nierlich, D. P., Roe, B. A., Sanger, F., Schreier, P. H., Smith, A. J., Staden, R. and Young, I. G. (1981) Nature, 290, 457-465.

26 Sambrook, J., Fritsch, E. F. and Maniatis, T. (1989) Molecular Cloning: A Laboratory Manual, 2nd Edn, Cold Spring Harbor University Press, Cold Spring Harbor, NY.

27 Shoffner, J. M., Fernhoff, P. M., Krawiecki, N. S., et al. (1992) Neurology, 42, 2168-2174.

28 Chung, S. S., Weindruch, R., Schwarze, S. R., McKenzie, D. I. and Aiken, J. M. (1994) Aging, 6, 193-200.

29 Gadaleta, M. N., Rainaldi, G., Lezza, A. M., Milella, F., Fracasso, F. and Cantatore, P. (1992) Mutat. Res, 275, 181-193.

30 Lee, C. M., Chung, S. S., Kaczkowski, J. M., Weindruch, R. and Aiken, J. M. (1993) J. Gerontol., 48, B201-B205.

31 Melov, S., Lithgow, G. J., Fischer, D. R., Tedesco, P. M. and Johnson, T. E. (1995) Nucleic Acids Res., 23, 1419-1425.

32 Shigenaga, M. K., Hagen, T. M. and Ames, B. N. (1994) Proc. Natl Acad. Sci. USA, 91, $10771-10778$.

33 Richter, C. (1988) FEBS Lett., 241, 1-5.

34 Bandy, B. and Davison, A. J. (1990) Free Radic. Biol. Med., 8, 523-539 